\title{
Higgs Physics at the LHC
}

\author{
Chiara Mariotti ${ }^{* \dagger}$ \\ INFN Torino (IT) \\ E-mail: chiara.mariottiecern.ch
}

The first measurements of the mass, the width, and the couplings of the newly discovered Higgs boson at LHC at 7 and $8 \mathrm{TeV}$ center of mass energy will be reviewed. Recent results at $13 \mathrm{TeV}$ center of mass energy will be presented. Finally, searches for additional Higgs bosons in models beyond the standard model will be summarised.

25th International Workshop on Deep Inelastic Scattering and Related Topics

3-7 April 2017

University of Birmingham, Birmingham, UK

*Speaker.

${ }^{\dagger}$ On behalf of the ATLAS and CMS experiments 


\section{Introduction}

A new particle compatible within the experimental uncertainty to be a Higgs boson has been discovered in 2012 by the ATLAS and CMS experiments [1,2] at the LHC accelerator at CERN. It has been a long search carried out by both the theoretical and the experimental communities, that has lasted more than 60 years. It is possible to identify the first foundation of the Standard Model (SM) of particle physics in the year 1954, when Yang and Mills defined the Gauge Theories to describe the interactions between massless particles and the gauge fields. The theory is renormalisable. In 1961 Glashow made the hypothesis that the electromagnetic interaction and the weak interaction are of the same kind, but the propagators that mediate the interaction are massless for the electromagnetic one and very massive for the weak one. In 1964 Brout, Englert and Higgs developed a mechanism thanks to which from one massless boson and two scalar particles, we can get one massive boson and one massive scalar. In 1967-1968 Weinberg and Salam combined the Yang and Mills theory, the Higgs mechanism and the hypothesis of Glashow and developed the Standard Model of electroweak interactions. In 1970 t'Hooft demonstrated that the theory is renormalisable. Right at that time at CERN, neutrinos beams were becoming operational, and in 1973 the experiment Gargamelle discovered the existence of neutral current, thus giving the first experimental indirect evidence of the existence of the $\mathrm{Z}$ boson, and thus a first estimate of $\sin \theta_{W}$. Since the value of $\sin \theta_{W}$ was suggesting the $\mathrm{W}$ and $\mathrm{Z}$ bosons mass to be around $100 \mathrm{GeV}$, Carlo Rubbia had the idea and managed to get the $S p \bar{p} S$ constructed at CERN, and in 1983 the UA1 and UA2 experiments discovered the $\mathrm{W}$ and $\mathrm{Z}$ boson, and measured their masses. In the years 1989 - 2000, the LEP machine with its 4 experiments at CERN and the HERA machine at DESY with its experiments marked the triumph of the SM. In the meantime, in 1995, CDF and D0 at the Tevatron at FNAL discovered the top quark. It was only in 2012 that finally the ATLAS and CMS experiments at LHC discovered a Higgs boson particle.

\section{Higgs boson phenomenology}

In the Standard Model (SM) when the electroweak symmetry is broken via the so-called BroutEnglert-Higgs mechanism (BEH) [3, 4, 5, 6, 7], vector bosons and fermions acquire mass and a new elementary particle with spin zero and positive parity appears: the Higgs boson. The ATLAS and CMS collaborations (the two general-purpose experiments at LHC $[8,9]$ ) announced in July 2012 the observation of a new resonance in diphoton and 4-leptons final states with a mass around $125 \mathrm{GeV}$, whose properties are, to date, compatible within the uncertainties with the Higgs boson predicted by the SM [1,2].

The Higgs boson production and decay rates measured by ATLAS and CMS give a combined signal yield, relative to the Standard Model (SM) prediction, of $1.09 \pm 0.11$ [26]. The Higgs boson mass is very precisely measured, and several decay modes have been observed with high significance. Gluon fusion and vector-boson fusion production modes have been observed, VH and $\overline{\mathrm{t}} \mathrm{HH}$ are not too far to reach the sensitivity to be observed.

The early discovery is certainly based on two pillars: experimental analysis improvements and theory accuracy improvements. To understand how they conspired to allow for the Higgs discovery see Refs. [11, 12]. 
The SM Higgs boson production cross sections and decay branching fractions have been calculated in the recent years at high order in perturbation theory. Many calculations have been compared and then eventually combined and summarised in Refs. [11, 12, 13, 14], and they are shown in Fig. 1 and in Tab. 1. The order of each of the calculations is shown in the table together with the uncertainty coming from the PDF and the QCD scale variations at $13 \mathrm{TeV}$ center of mass energy.

Table 1: Higgs boson production processes for $m_{\mathrm{H}}=125.09 \mathrm{GeV}$ at $13 \mathrm{TeV}$ center of mass energy.

\begin{tabular}{llcc}
\hline \hline production process & order of calculations & Scale unc. (\%) & PDF unc. (\%) \\
$\mathrm{gg} \rightarrow \mathrm{H}$ & NNNL QCD, NLO EW & 4 & 1.9 \\
$\mathrm{qq} \rightarrow \mathrm{qqH}$ & NNLO QCD, NLO EW & 0.4 & 2.1 \\
$\mathrm{qq} \rightarrow \mathrm{WH}$ & NNLO QCD, NLO EW & 0.5 & 1.7 \\
$\mathrm{qq} \rightarrow \mathrm{ZH}$ & NNLO QCD, NLO EW & 3.5 & 1.3 \\
$\mathrm{qq}, \mathrm{gg} \rightarrow \overline{\mathrm{t}} \mathrm{tH}$ & NLO QCD, NLO EW & 7 & 3 \\
$\mathrm{qq}, \mathrm{gg} \rightarrow \overline{\mathrm{b}} \mathrm{bH}$ & 5FS NNLO$(\mathrm{QCD})+4 \mathrm{FS}$ NLO $(\mathrm{QCD})$ & \multicolumn{2}{c}{ Tot. 20} \\
$\mathrm{qg} \rightarrow \mathrm{tH}$ & NLO QCD & 10 & 3.5 \\
\hline \hline
\end{tabular}
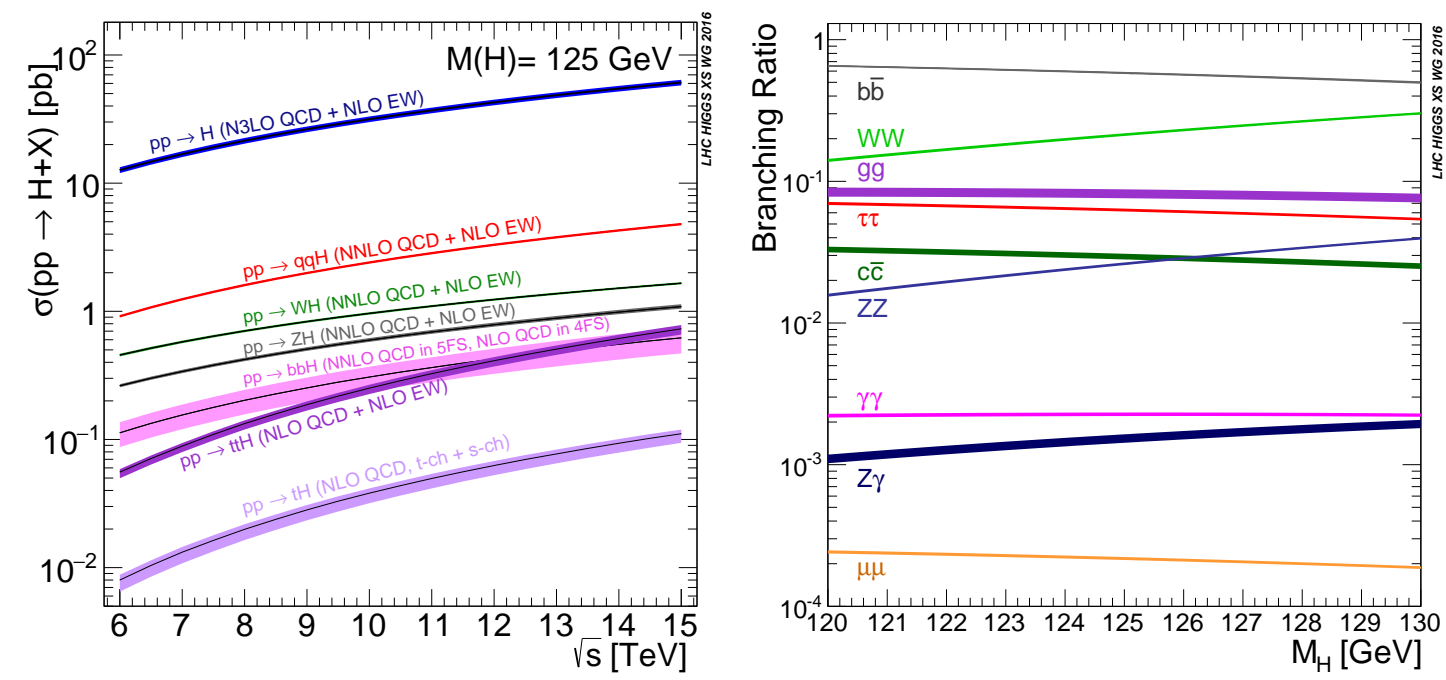

Figure 1: Standard Model Higgs production cross sections for $m_{\mathrm{H}}=125 \mathrm{GeV}$ as a function of the center of mass energy and branching ratios.

\section{The measurement of the mass}

The most important parameter of a particle is its mass, although the definition of mass and width for an unstable particle requires particular care and is not unique. Here we discuss on-shell quantities.

The Higgs boson mass can be measured with very high precision from the $\mathrm{H} \rightarrow 41$ and $\mathrm{H} \rightarrow$ $\gamma \gamma$ decays, since muons, electrons and photons are reconstructed with high precision, see Refs. $[15,16]$.

The energy scale, the momentum scale and resolution of muons, electrons and photons are excellent in both the experiments. Well-known particles like the $\mathrm{Z}, \mathrm{Y}, \mathrm{J} / \psi \rightarrow 21$ are used to calibrate 
the detectors. The decay $\mathrm{Z} \rightarrow 4 \mathrm{l}$ is used to validate the procedure. The systematic uncertainty is $\pm 0.1 \% / \pm 0.3 \%$ for the muon/electron momentum scale in CMS, and $\pm 0.3 \% / \pm 0.1 \%$ for the muon/electron momentum scale in ATLAS. In CMS the mass measurement is performed with a 3D fit using four-lepton invariant mass $m_{41}$, associated per-event mass uncertainty $\delta m_{41}$, and kinematic discriminant $K D$, see Ref. [17].

The photon energy calibration is the dominant systematic uncertainty in the mass reconstruction for the $\mathrm{H} \rightarrow \gamma \gamma$ channel. The energy scale is determined using $\mathrm{Z} \rightarrow \mathrm{e}^{-} \mathrm{e}^{+}$events, then a correction is applied to account for the $\mathrm{e}-\gamma$ difference and subsequently an extrapolation is performed in order to move from the energy scale of the $\mathrm{Z}$ to the energy scale of the $\mathrm{H}$. In summary, the systematic uncertainties in the mass measurement from the $\gamma \gamma$ channel are due to the knowledge of the material in front of the electromagnetic calorimeter, the non-linearity of the calorimeter, the calibration of the detector, and the differences between electron and photons.

The mass of the Higgs boson as measured from the first run at LHC at 7 and $8 \mathrm{TeV}$ center of mass energy is $[15,16,18]$ :

$$
m_{\mathrm{H}}=125.09 \pm 0.24 \mathrm{GeV}=125.09 \pm 0.21(\text { stat }) \pm 0.11(\mathrm{syst}) \mathrm{GeV}
$$

where the total uncertainty is dominated by the statistical term, with the systematic uncertainty dominated by the non linearity of the electromagnetic calorimeter and by the knowledge of the material in front of them and by the lepton energy/momentum scale uncertainty.

\section{The Higgs boson width}

In Ref. [19] the off-shell production cross section has been shown to be sizeable at high 41invariant mass in the gluon fusion production mode, with a ratio relative to the on-peak cross section of the order of $8 \%$ at a center-of-mass energy of $8 \mathrm{TeV}$. This ratio can be enhanced up to about $20 \%$ when a kinematical selection used to extract the signal in the resonant region is taken into account [20]. This arises from the vicinity of the on-shell $\mathrm{Z}$ pair production threshold, and is further enhanced at the on-shell top pair production threshold.

In Ref. [21] the authors demonstrated that, with few assumptions and using events with pairs of $\mathrm{Z}$ particles, the high invariant mass tail can be used to constrain the Higgs width. For a detailed description, see Ref. [22]. The cross section for the on-shell production will not change if the squared product of the coupling constants $g_{\mathrm{ggH}}^{2} g_{\mathrm{HZZ}}^{2}$ and the total width $\Gamma_{\mathrm{H}}$ are scaled by a common factor $r$. On the contrary, away from the resonance the cross section is independent of the total width and therefore increases linearly with $r$. Thus a measurement of the relative off-shell to onshell production in the $\mathrm{H} \rightarrow \mathrm{ZZ}$ channel provides direct information on $\Gamma_{\mathrm{H}}$, as long as the coupling ratios remain unchanged, i.e. the gluon fusion production is dominated by the top-quark loop and there are no new particles contributing.

The final states $\mathrm{H} \rightarrow \mathrm{ZZ} \rightarrow 4 \mathrm{l}$, where the $\mathrm{Z}$ bosons decay to an e or $\mu$ pair, $\mathrm{H} \rightarrow \mathrm{ZZ} \rightarrow 212 v$ and $\mathrm{H} \rightarrow \mathrm{WW} \rightarrow 212 v$ have been analysed in ATLAS [23], and CMS [24, 25].

The observed 95\% CL upper limits on the $\mathrm{H}$ width from the analysis of the off-shell $\mathrm{H}$ production for the ATLAS and CMS experiments are 22.7 and $13 \mathrm{MeV}$ for ATLAS and CMS respectively, while the expected 95\% CL upper limits are $33 \mathrm{MeV}$ and $26 \mathrm{MeV}$. 


\section{The Higgs boson couplings}

The signal strengths $\mu_{i}^{f}$ are defined as the ratios of cross sections and branching fractions to the corresponding SM predictions such that:

$$
\mu_{i}^{f}=\frac{\sigma_{i} \cdot B R^{f}}{\left(\sigma_{i}\right)_{\mathrm{SM}} \cdot\left(B R^{f}\right)_{\mathrm{SM}}}=\mu_{i} \times \mu^{f},
$$

where the subscript $i$ and superscript $f$ indicate the production mode and decay channel, respectively. By definition all $\mu_{i}^{f}$ are equal to 1 for the SM Higgs boson.

The simplest and most restrictive signal strength parameterisation is to assume that the $\mu_{i}$ and the $\mu^{f}$ values are the same for all production processes and decay channels. In this case, the SM predictions of signal yields in all categories are scaled by a global signal strength $\mu$. Such a parameterisation provides the simplest test of the compatibility of the experimental data with the SM predictions.

A fit to the combined ATLAS and CMS data at $E_{\mathrm{CM}}=7,8 \mathrm{TeV}$, with $\mu$ as the parameter of interest, results in the best-fit value:

$$
\left.\mu=1.09_{-0.10}^{+0.11}=1.09 \pm 0.07(\text { stat }) \pm 0.04(\text { expt }) \pm 0.03 \text { (th-bkgd }\right)_{-0.06}^{+0.07}(\text { th-sig })
$$

As a further step we can measure signal strengths for the different production modes and decays modes. The production processes can be divided into two subgroups: the production via strong interactions where the coupling is a fermion coupling, or the production via EW production where the coupling is a vector boson coupling. The result is shown in Fig. 2 left..
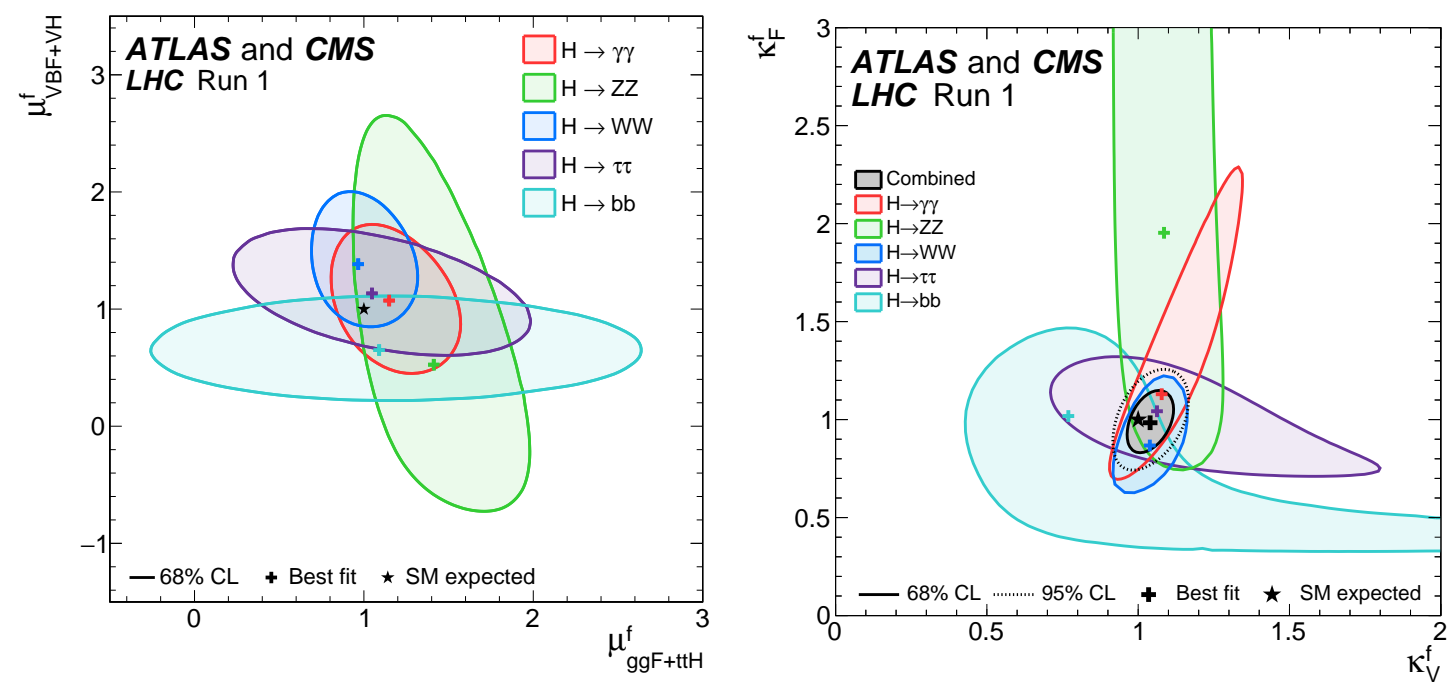

Figure 2: Left: Negative log-likelihood contours at $68 \% \mathrm{CL}$ in the $\left(\mu_{g g F+t t H}, \mu_{V B F+V H}\right)$ plane for the combination of ATLAS and CMS, for each of the final states analysed: $\mathrm{H} \rightarrow \mathrm{ZZ}, \mathrm{H} \rightarrow \mathrm{WW}, \mathrm{H} \rightarrow \gamma \gamma$, $\mathrm{H} \rightarrow \tau \tau, \mathrm{H} \rightarrow \mathrm{bb}$, and their combination. The SM expectation is also shown as a black star. The figure is from Ref. [26]. Right: Negative log-likelihood contours at $68 \% \mathrm{CL}$ in the $\left(\mathrm{\kappa}_{\mathrm{f}}, \mathrm{\kappa}_{\mathrm{V}}\right)$ plane for the combination of ATLAS and CMS and for the individual decay channels as well as for their global combination, assuming that all coupling modifiers are positive. The figure is from Ref. [26]. 
Vector and fermion Higgs couplings in the kappa framework. The further step is to assume that all fermion couplings scale as $\kappa_{\mathrm{f}}$ while all vector boson couplings scale as $\kappa_{\mathrm{V}}$. The result is shown in Fig. 2-right. The figures shows the 5 different final states and their combination.

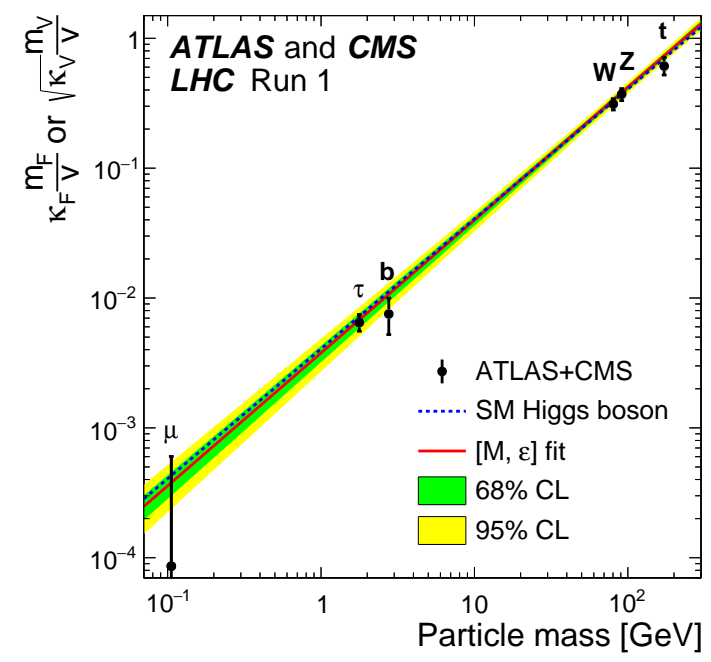

Figure 3: Fit results as a function of the particle mass in the case of the parameterisation with reduced coupling modifiers $y_{V, i}$ for the weak vector bosons, and $y_{f, i}$ for the fermions, as explained in the text, for the combination of ATLAS and CMS. The dashed line indicates the predicted dependence on the particle mass for the SM Higgs boson. The solid (red) line indicates the best fit result to the $[\mathrm{M}, \varepsilon]$ phenomenological model of Ref. [27] with the corresponding 68\% and 95\% CL bands in green and yellow. The figure is from Ref. [26].

The relation between the coupling modifiers and the SM predictions can be presented as a function of the mass of the particles to which the $\mathrm{H}$ boson is coupling. The coupling of the Higgs to vector bosons of mass $m_{\mathrm{V}}$ when expressed as a function on $\kappa_{\mathrm{V}}$ is:

$$
y_{V, i}=\sqrt{\kappa_{\mathrm{V}, i} \frac{g_{\mathrm{V}, i}}{2 v}}=\sqrt{\kappa_{\mathrm{V}, i}} \frac{m_{\mathrm{V}, i}}{v}
$$

where $g_{\mathrm{V}, i}$ is the absolute Higgs boson coupling strength and $v=246 \mathrm{GeV}$ is the vacuum expectation value of the Higgs field.

The coupling of the Higgs to the fermions of mass $m_{\mathrm{f}}$ when expressed as a function on $\kappa_{\mathrm{f}}$ is:

$$
y_{f, i}=\kappa_{\mathrm{f}, i} \frac{g_{\mathrm{f}, i}}{\sqrt{2}}=\kappa_{\mathrm{f}, i} \frac{m_{\mathrm{f}, i}}{v} .
$$

The linear scaling of the reduced coupling modifiers as a function of the particle masses is shown in Fig. 3, left and indicates the consistency of the measurements with the SM.

\section{New results at $13 \mathrm{TeV}$}

When the center of mass energy raises from 8 to $13 \mathrm{TeV}$ the cross section for the gluon-gluon fusion, for the Vector Boson Fusion and for the associated production with a $\mathrm{W}$ or a $\mathrm{Z}$ become 
about 2 times larger. For the associated production with a pair of top quarks the cross section instead raises of about a factor of 4 (see Fig. 1). The ATLAS and the CMS experiments have collected about $36 \mathrm{fb}^{-1}$ at $13 \mathrm{TeV}$ in the year 2016 .

Combined ATLAS and CMS results on $\overline{\mathrm{t}} \mathrm{tH}$ production based on the LHC Run 1 data set showed an intriguing excess: the measured rate was above the SM prediction with a statistical significance corresponding to $2.3 \sigma$. The latest results of $\mathrm{ttH}$ searches at CMS presented at the winter 2017 conferences, show that the experiments are on the verge of measuring this crucial process with sufficient precision to confirm or disprove the previous observed excess. With a larger data set it should be possible to have clear evidence for $\overline{\mathrm{t}} \mathrm{tH}$ production by the end of Run 2 .

The experiments could perform many studies and provide many results with the $\mathrm{H} \rightarrow 41$ and $\mathrm{H} \rightarrow \gamma \gamma$ decays [28, 29, 30, 31]. Figure 4 show the mass spectra for ATLAS and CMS. CMS measures the mass of the Higgs boson with the $\mathrm{H} \rightarrow 4 \mathrm{l}$ decays only, with a precision better than the Run 1 result: $m_{\mathrm{H}}=125.26 \pm 0.20$ (stat.) \pm 0.08 (syst.) GeV. Differential and total cross sections shows good agreement with the theoretical predictions, within the uncertainty.

The results of the search for $\mathrm{H} \rightarrow \mu \mu$ in ATLAS [32] are shown in Fig. 5 right. The measured signal strength $\mu$ is $-0.07 \pm 1.5$ at $13 \mathrm{TeV}$ and $-0.13 \pm 1.4$ for the combined $7+8+13 \mathrm{TeV}$ data with an observed (expected) limit on the signal strength of 2.8 (2.9) at 95\%CL .

With the large statistic at $13 \mathrm{TeV}$ many searches for the production of two Higgs bosons have been performed. In the SM the measurement of $\sigma_{\mathrm{HH}}$ allows to measure the Higgs trilinear coupling $\lambda_{\mathrm{HHH}}$. The cross section is very small due to the destructive interference of the two leading-order diagrams of double Higgs production. Beyond the standard model (BSM) the HH production can be resonant, thus an extra Higgs boson or a new particle can decay into two Higgs bosons of 125 $\mathrm{GeV}$ of mass. The searches can go up to a resonant invariant mass of $3000 \mathrm{GeV}$. Up to now only upper limits have been set, and no signal has been observed.

\section{Is this the SM Higgs boson?}

The ATLAS and CMS experiments have discovered a scalar particle. Its spin parity has been determined to be $0^{+}$at more than $99.9 \%$ confidence level $[33,34]$. The signal strengths are all compatible with 1 within $10 \%$ to $30 \%$ : the ggF production is well established, the VBF is at $5.4 \sigma$, the $\mathrm{WH}+\mathrm{ZH}$ is at $3 \sigma$. We are not yet sensitive to observe the $\overline{\mathrm{t}} \mathrm{H}$ production process. The $\mathrm{ZZ}$, WW, $\gamma \gamma$ decays are well established, the decay to $\tau \tau$ is at $5 \sigma$ when combining ATLAS and CMS. There is not yet evidence of the decay into b quarks.

The most interesting question is to understand if this boson is the SM Higgs boson, or it is one of the Higgs bosons of the many models that predict new physics. We have to determine whether this Higgs particle is fully responsible for the generation of the masses of the other SM particles and whether it fully unitarises the high-energy scattering amplitudes.

There are two ways to attack this problem: one is to look for new particles, i.e. for other Higgs bosons or for non-SM decay of this newly discovered particle; the other is to measure with high precision the properties of this particle and the di-boson scattering cross section.

The simplest extension to the SM gives the possibility to have one additional Higgs boson, an electroweak (EW) singlet. A more complex extension allows the existence of a second doublet of Higgs fields. The general 2HDM (2 Higgs doublet model), that does not imply supersymmetric 

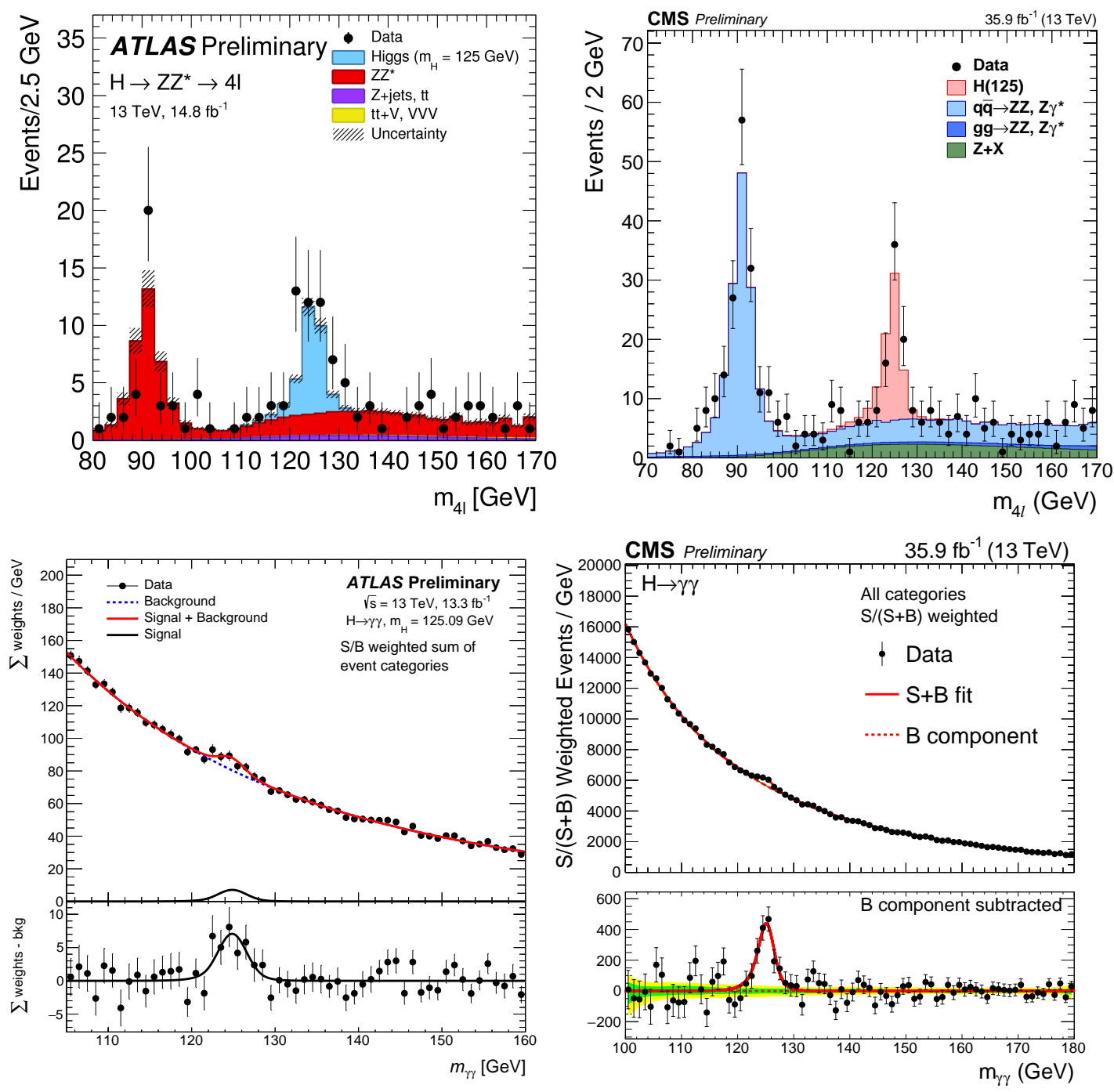

Figure 4: Top: four leptons invariant mass spectra for ATLAS (left) and CMS (right) at $13 \mathrm{TeV}$. Bottom: two photons invariant mass spectra for ATLAS (left) and CMS (right) at $13 \mathrm{TeV}$. The figures are from Refs. [28, 29, 30, 31].

particles, the MSSM (minimal supersymmetric model), and the fermiophobic model are studied carefully, as well as the nMSSM model that predicts two Higgs doublets and one additional singlet. Finally more searches with final states that are rare in the SM but can be enhanced in case of new physics are also searched for at LHC. All these models have been studied in details and searches for the predicted particles have been carried on in the experiments. These searches have not given positive results and have excluded large regions of the parameter space.

The possibility to measure precisely the cross section of boson-boson scattering as a function of the invariant mass of the di-bosons will be crucial to discriminate between various models of electroweak symmetry breaking, i.e. to determine at which scale and by which mechanism the high-energy scattering amplitudes are unitarised. This argument has not been covered in this talk.

Ultimately the precision measurements of the Higgs properties, its couplings and quantum 


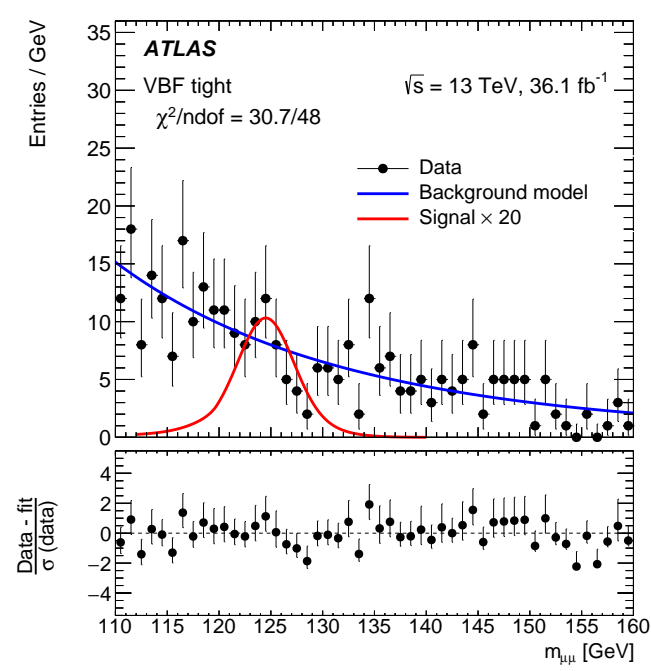

Figure 5: Two muons invariant mass spectra in the ATLAS experiment at $13 \mathrm{TeV}$. The red curve is the expected signal multiplied by a factor 20 . The figure is from Ref. [32]

numbers, will shed light into the possibility of new physics beyond the SM.

\section{Summary}

The discovery of the Higgs boson in 2012 at LHC has been sensational from many points of view: it was the first ever elementary scalar particle discovered, it was discovered very soon after the start-up of LHC thanks to the impressive performance of the machine, and it was the last missing piece of the SM. On the other side, its mass is right at the limit to make our universe meta-stable. The mass value does not explain the naturalness, nor the mass hierarchy, thus leaving many unanswered questions and creating many more. As Gödel's incompleteness theorems say: you cannot get an ultimate answer, since every answer creates automatically a new question. Yariv Friedman rephrase it : "the act of discovery is basically what creates the unknown" [35].

\section{References}

[1] ATLAS Collaboration (G. Aad et al.), Phys. Lett. B 716, 1 (2012).

[2] CMS Collaboration (S. Chatrchyan et al.), Phys. Lett. B 716, 30 (2012).

[3] F. Englert and R. Brout, Phys. Rev. Lett. 13, 321 (1964).

[4] P. W. Higgs, Phys. Rev. Lett. 13, 508 (1964).

[5] G. Guralnik, C. Hagen and T. Kibble, Phys. Rev. Lett. 13, 585 (1964).

[6] P. W. Higgs, Phys. Rev. 145, 1156 (1966).

[7] T. Kibble, Phys. Rev. 155, 1554 (1967).

[8] ATLAS Collaboration (G. Aad et al.), JINST 3 S08003 (2008)

[9] CMS Collaboration (S. Chatrchyan et al.), JINST 3 S08004 (2008) 
[10] ATLAS, CMS Collaboration (G. Aad et al.), JHEP 08, 045 (2016).

[11] LHC Higgs Cross Section Working Group Collaboration (S. Dittmaier et al.) (2011), arXiv:1101.0593 [hep-ph], CERN-2011-002.

[12] LHC Higgs Cross Section Working Group Collaboration (S. Dittmaier et al. (2012), arXiv:1201.3084 [hep-ph], CERN-2012-002.

[13] LHC Higgs Cross Section Working Group Collaboration (S. Heinemeyer et al.) (2013), arXiv:1307.1347 [hep-ph], 10.5170/CERN-2013-004.

[14] LHC Higgs Cross Section Working Group Collaboration (de Florian, D. et al.) (2016) arXiv:1610.07922 [hep-ph], CERN-2017-002-M.

[15] ATLAS Collaboration (G. Aad et al.), Phys. Rev. D 90, 052004 (2014)

[16] CMS Collaboration (V. Khachatryan et al.), Eur. Phys. J. C 75, 212 (2015)

[17] CMS Collaboration, Phys. Rev. D 89, 092007 (2014)

[18] ATLAS, CMS Collaborations (G. Aad et al.), Phys. Rev. Lett. 114, 191803 (2015)

[19] N. Kauer and G. Passarino, JHEP 08, 116 (2012)

[20] N. Kauer, Mod.Phys.Lett. A 28, 1330015 (2013)

[21] F. Caola and K. Melnikov, Phys.Rev. D 88, 054024 (2013)

[22] G. Passarino, Eur. Phys. J. C 74, 2866 (2014)

[23] ATLAS Collaboration (G. Aad et al.), Eur. Phys. J. C 75, 335 (2015)

[24] CMS Collaboration (V. Khachatryan et al.), Phys. Lett. B 736, 64 (2014)

[25] CMS Collaboration (V. Khachatryan et al.), JHEP 09, 051 (2016)

[26] ATLAS, CMS Collaboration (G. Aad et al.), JHEP 08, 045 (2016)

[27] J. Ellis and T. You, JHEP 06, 103 (2013).

[28] ATLAS Collaboration, Conference note ATLAS-CONF-2016-079

[29] CMS Collaboration, Public note CMS-HIG-16-041

[30] ATLAS Collaboration, Conference note ATLAS-CONF-2017-067

[31] CMS Collaboration, Public note CMS-HIG-17-015

[32] ATLAS Collaboration, Conference note ATLAS-CONF-2017-014

[33] ATLAS Collaboration, ( G. Aad et al.), Eur. Phys. J. C 75, no. 10, 476 (2015) Erratum: [Eur. Phys. J. C 76, no. 3, 152 (2016)]

[34] CMS Collaboration (V. Khachatryan et al. ) Phys. Rev. D 92 no. 1, 012004 (2015)

[35] http://geneva.impacthub.net/member-story-an-inquisitive-mind/ 